\title{
JUSTIFICACIÓN DE LAS OBLIGACIONES EN LA JUSTICIA INTERGENERACIONAL
}

\author{
Justification of Obligations in Intergenerational Justice \\ José Manuel Gragera Junco* \\ Universidad de Málaga \\ chemagj@hotmail.es
}

\begin{abstract}
Resumen:
En este artículo haré una revisión de las distintas teorías de la justicia intergeneracional. Para este fin, analizaré en virtud de qué principios surgen las obligaciones entre generaciones para las distintas teorías. Además de buscar la justificación de tales deberes intergeneracionales el análisis irá dirigido a mostrar cuál es el contenido de las obligaciones que cada una de las teorías exige. En otras palabras, este trabajo tiene el propósito de examinar qué justifica para cada teoría que existan obligaciones entre generaciones y cuál es la medida de reparto en que esos deberes deben establecerse. Finalmente, argumentaré en favor de la teoría de justicia intergeneracional que considero superior normativamente.
\end{abstract}

\section{Palabras clave:}

Justicia intergeneracional, obligaciones, justificación, distribución.

\begin{abstract}
:
In this paper, I shall review the different theories of intergenerational justice. For this aim, I will analyze by virtue of which principles arise the obligations between generations for the different theories. Besides looking for the justification of such intergenerational duties, the analysis will be aimed at showing what is the content of obligations that each theory demands. In other words, this work has the purpose of examining what justifies for each theory that there are obligations between generations and what is the measure of distribution in which those duties must be established. Finally, I will argue in favor of the theory of intergenerational justice that I consider normatively superior.
\end{abstract}

\section{Keywords:}

Intergenerational justice, Obligations, Justification, Distribution.

\footnotetext{
* Agradezco a Manuel Toscano su inestimable ayuda y su constante guía para que este trabajo saliera adelante.
} 


\section{INTRODUCCIÓN}

Una adecuada concepción de la justicia intergeneracional tiene la capacidad de establecer un sistema relacional en el que distintas generaciones están sujetas a una estructura normativa definida en términos de derechos y obligaciones. Indagar acerca de cuáles son las razones subyacentes a estos correlativos y opuestos constituye una manera pertinente de acercarnos a una intuición de lo que es considerado como justo.

El debate intergeneracional de la justicia suscita preguntas muy diferentes. ¿Debemos hacer todo lo posible por afianzar el futuro de las generaciones que nos sucederán? ¿O acaso no disponen ya, sin ni siquiera haber nacido, de una herencia lo suficientemente grande? Pero esencialmente, ¿por qué hemos de tener la obligación de transferir nada a estas generaciones futuras? ¿En virtud de qué? Si bien la justicia entre generaciones implica incluir en nuestros procesos deliberativos los posibles intereses de aquellas generaciones que no tendrán ninguna transcendencia en nuestras vidas, acoger una determinada concepción de lo que consideremos como justo en materia intergeneracional sí tiene el potencial de dotarnos de herramientas para transformar de manera notable nuestro modo de vida. Bien sea en ámbitos como la sostenibilidad medioambiental, la sostenibilidad y justicia de un sistema público de pensiones o readaptación de nuestra vida a la creciente longevidad experimentada en las últimas décadas.

Por ello, mi trabajo consistirá en arrojar algo de luz sobre los posibles motivos que nos inducirían a sostener que tenemos ciertas obligaciones con el resto de generaciones, especialmente las futuras aún no nacidas. Adicionalmente incidiré en la medida distributiva que cada una de esas razones justificadoras nos imponen con el expreso fin de constatar por qué debemos algo al respecto de generaciones con las que ni siquiera vamos a convivir.

\section{JUSTICIA COMO RECIPROCIDAD INDIRECTA}

La primera teoría de la justicia intergeneracional que voy a tratar es la teoría de la reciprocidad indirecta (Gosseries, 2001: 297-303; 2004: 148-183; 2005: 41-42; 2015: 222-224). El análisis de los términos que la componen puede ofrecer un primer indicio de lo que significa e implica suscribir una teoría como esta. En primer lugar, el término reciprocidad hace alusión a una suerte de retorno de algo previamente dado por otro individuo, de modo que sólo existe reciprocidad en la medida en que se devuelva algo a cambio. Es la idea que subyace en el acto de devolver lo prestado o concedido.

No se habla aquí de ofrecer o proporcionar algo a alguien sin esperar nada en cambio, sino que es precisamente ese "algo a cambio" o en retorno lo que caracteriza a la reciprocidad. Además, la idea de la reciprocidad no sólo sugiere una transacción entre dos partes como en las habituales de arrendador y arrendatario, sino que sitúa el foco y la carga de la prueba en la persona a la que se le exige la reciprocidad: el 
deudor. De tal manera, aunque la reciprocidad sólo es posible en virtud de dos partes que establecen por mutuo acuerdo y mutuo beneficio algún tipo de actividad o transacción, recae sobre la segunda persona la obligación de dar cumplimiento a dicha relación recíproca. Es decir, si lo que exige la reciprocidad es la existencia de al menos dos partes que se procuran una determinada correspondencia en virtud de una actividad convenida, también exige que la obligación de dar sentido a esa correspondencia se encuentre en la respuesta de la segunda parte. En otras palabras: sin deudor no hay reciprocidad posible. De no darse la figura de alguien que debe nos encontraríamos simplemente en una situación en la que alguien ofrece o regala algo a alguien sin que exista la obligación de un retorno.

Por otro lado, el adjetivo indirecto que sigue a la reciprocidad nos señala la dirección de la obligación. Si la reciprocidad nos habla de en qué se fundamenta la obligación (deuda contraída por el préstamo o el favor de alguien), el término indirecto marca la dirección en la que ha de solventarse esa deuda. Aquí es donde tiene sentido hablar del ámbito intergeneracional. Si una reciprocidad directa implica la devolución a quien presta, una de tipo indirecto no puede sino situar la devolución en otra parte diferente al acreedor: es decir, en un tercer participante del pacto recíproco. De hecho, los sistemas de pensiones por reparto, como el español, se asientan en el marco de este presupuesto indirecto. Lo que aporta la generación trabajadora $(\mathrm{G} 2)^{1}$ para pagar las pensiones no se lo devuelven los jubilados (G1) que reciben la pensión de manera directa, sino los futuros trabajadores (G3) en el momento de entrar en el mercado laboral siendo, entonces, G1 la generación actual jubilada. El marco intergeneracional plantea ciertas complicaciones al respecto. En este sentido, estaríamos hablando de "pagar una deuda" a una generación que es distinta a la generación acreedora. Es más, hablaríamos de pagar dicha deuda a una generación que, probablemente, no va a reportar ningún tipo de beneficio a la acreedora y con la cual puede no convivir ni coexistir.

Siendo así las cosas, disponemos de elementos de juicio para dilucidar qué requiere y bajo qué términos se establece esta teoría de la justicia intergeneracional. Nos encontramos ante un marco teórico que exige que cada una de las generaciones devuelvan a otra generación lo previamente heredado o percibido. Lo que reciba la segunda generación (G2) de la primera generación (G1) debe retornarlo de manera indirecta, esto es, a la tercera generación (G3). En otras palabras, G2 transferirá algo a G3 por haber heredado algo de G1 (Gosseries, 2001: 299). Ahora bien, lo que intuitivamente podríamos reclamar en un pacto de este tipo es una devolución que, si bien no sea exactamente igual a la cantidad prestada, al menos sí sea equivalente ${ }^{2}$.

\footnotetext{
1 Por abreviación en la escritura, usaré G1, G2 y G3 (en referencia a la traducción inglesa) para referir a cada una de las generaciones.

${ }^{2}$ En última instancia, la reciprocidad sólo es posible en virtud de que se produzca una cierta simetría o igualdad, pues en la medida en que las partes estén desniveladas resulta ciertamente difícil poder articular una verdadera reciprocidad. Un claro ejemplo sería una relación esclavo-señor. La elevada posición del señor sobre el esclavo y, especialmente, la ausencia de derechos y libertades que amparen a este último, difícilmente harán que las deudas contraídas por el esclavo sean justas.
} 
Por tanto, debe existir una determinada proporcionalidad entre las partes y las transferencias acordadas para que el pacto recíproco pueda garantizarse.

Lo que indica esta proporcionalidad en las transferencias puede definirse como el contenido de nuestras obligaciones o, por decirlo de otra forma, la medida con la cuál establecer la cantidad que debemos a otras generaciones. De esta manera, tendríamos los dos principios vertebradores de la reciprocidad indirecta: la máxima justificadora y la máxima sustantiva. Mientras que la primera ofrece la justificación de la existencia de nuestras obligaciones (transferir alguna cosa a la generación siguiente por haber recibido algo de la generación precedente), la segunda establece una definición para el contenido de estos deberes: a saber, transferir a la siguiente generación al menos tanto como lo recibido por la generación previa a la nuestra (Gosseries, 2004: 149$150)^{3}$.

Sin embargo, la teoría de la reciprocidad cuenta con algunas deficiencias que la convierten en una teoría de la justicia intergeneracional frágil. Esta fragilidad se cifra en, al menos, tres objeciones. Primero, esta teoría debe hacer frente al inconveniente que nace de las transferencias excesivamente bajas respecto a la cantidad inicial prestada (Gosseries, ibid.: 150-152) y que supone un desafío a la máxima sustantiva. Es decir, la cantidad que hereda la generación inmediatamente anterior a la nuestra. Es esta la situación en la que una generación transfiere menos de lo que ella misma heredó. En este caso, G2 dona a G3 una cantidad muy por debajo de lo que ella heredó de G1. Si nos atenemos a la máxima sustantiva, ésta establece que la deuda de G2 con G3 sea, al menos, equivalente a la cantidad que recibió G" de G1. Pero si esta cantidad resulta ser muy inferior a la herencia de la primera transferencia intergeneracional (de G1 a G2), ¿qué debería hacer G3 cuando tenga que afrontar una transferencia a la cuarta generación? Según la máxima sustantiva no tiene la obligación de "apretarse el cinturón" para transferir más de lo recibido, pero es de dudosa justicia que los derechos de G4 puedan verse comprometidos por culpa de las ineficiencias de las generaciones anteriores. Esta situación también deja gravemente comprometida a la generación deudora (G3, aquí), haciendo recaer sobre ella todo el peso en caso de verse obligada a aumentar la cesta de transferencias a su cantidad inicial. De modo que devolver la cantidad a transferir a su cuantía inicial puede suponer una medida injusta para la generación deudora y no hacerlo es injusto con la que recibirá.

El segundo problema procede de la obligación que surge a través de los regalos (gift-obligation), señalado por Brian Barry (1989a). Brevemente, este planteamiento señalaría la difícil justificación que podría ofrecer una teoría de la reciprocidad al hecho de derivar las obligaciones de algo que se concede en calidad de regalado. Dicho de otra forma, la objeción ataca directamente a la máxima justificadora cues-

\footnotetext{
${ }^{3}$ Es importante no confundir la equivalencia en las transferencias con tomar como modelo de desarrollo el utilizado por las generaciones anteriores. El punto de referencia es la cantidad de la transferencia, no el esfuerzo para hacerla efectiva. No podemos tasar nuestra deuda con la siguiente generación en virtud del porcentaje de crecimiento en el PIB que hayan conseguido nuestros padres sólo porque sus circunstancias les haya obligado a gastar menos (Gosseries, ibid.: 151).
} 
tionando que de cada regalo que puedan hacerse las generaciones entre sí pueda surgir, de manera consistente, una obligación relativa que caiga del lado de aquellos que serán beneficiarios del mismo (Gosseries, 2001: 299-300; 2004: 161-162). En estos términos, la propia definición de "regalar" es esclarecedora: "dar a alguien, sin recibir nada a cambio, algo en muestra de afecto o consideración o por otro motivo" ${ }^{4}$. De manera que la propia definición de lo que un regalo implica se muestra abiertamente incompatible con la máxima justificadora desde el momento en que se establece que aquella cosa que será debida a la generación siguiente se deberá en virtud de haber sido previamente heredada o prestada por la generación anterior. Por tanto, una teoría de la reciprocidad -en su forma directa o indirecta- no puede justificar que surja una deuda a causa de un regalo recibido.

Finalmente, encontramos el argumento de la primera generación. Según esta crítica, no hay nada que obligue a la primera generación de todas a tener que transferir una cesta de recursos o bienes a la siguiente generación al no existir un préstamo anterior a ese (Gosseries, 2004: 178-181; 2005: 41-42; 2015: 223). Es la idea de que la primera generación de seres humanos no disponía de generaciones anteriores a ella misma para recibir algo y contraer así una deuda con las generaciones sucesoras, por lo que entra en conflicto con la máxima que justifica la existencia de obligaciones hacia generaciones posteriores ${ }^{5}$. A esta complejidad se le añade una no menos importante. En cierto sentido, buena parte de las generaciones que han existido podrían, incluso, ser consideradas como una hipotética primera generación si tenemos en cuenta los descubrimientos y avances científicos (Gosseries, 2001: 302-303). Es decir, una generación que, por definición, no ha recibido algo de la anterior: el primer viaje a la luna, el descubrimiento de la máquina de Turing o el bosón de Higgs, por ejemplo. Más allá de ser una circunstancia sin importancia, pone a prueba a la teoría. Si el surgimiento de las obligaciones procede de recibir algo de la generación anterior y tal obligación exige que lo que se ha heredado se transfiera a la siguiente generación, del caso en que algo determinado no haya sido heredado sino descubierto o desarrollado por la propia generación no se puede seguir que exista la obligación de transferir ese algo. O dicho de otra forma: la obligación de transferir a las siguientes generaciones sólo se dan en caso de haber recibido algo de la precedente, si algo no se obtiene en calidad de prestado no hay nada en la teoría que avale el deber de dejar esto mismo en herencia.

\footnotetext{
${ }^{4}$ Real Academia Española (2014). Disponible en: http://dle.rae.es/?id=Vgmi2Af|VgnZr1k Fecha de consulta: 21/10/2017.

${ }^{5}$ Esta dificultad se aprecia al intentar articular políticas públicas intergeneracionales como los sistemas de pensiones, donde la primera generación gozó de una pensión pública sin haber contribuido a un sistema público de pensiones (por no estar establecido previamente). Es lo que se ha llamado el free-lunch de las pensiones (Gosseries, 2004: 271-281; Schokkaert y van Parijs, 2003).
} 


\section{JUSTICIA COMO VENTAJA MUTUA}

La idea de la ventaja mutua como elemento central en una teoría de la justicia intergeneracional comparte cierto parecido con la de justicia entendida como reciprocidad. Se asemejan en la medida en que todas las partes en relación se prestan, usan y disfrutan aquellos bienes que decidan, pero se diferencian en que para una (reciprocidad) las obligaciones se heredan de transferencia en transferencia y ligan el contenido de sus obligaciones al contenido de sus pagos generacionales $y$, para la otra (ventaja mutua), dichas obligaciones son configuradas de acuerdo a su posible aceptabilidad por las generaciones en disputa (Fleurbaey y Michel, 1992: 51-54) habiendo tenido en cuenta -cada una de ellas- sus preferencias del momento. Por decirlo de manera más sencilla, la principal divergencia nace de la imposición y herencia de obligaciones (y su contenido) de una generación a la otra (reciprocidad), por un lado, y del mutuo acuerdo y negociación de éstas entre las distintas generaciones (ventaja mutua), por otro. Ahora bien, la consolidación y realización de una teoría de la justicia como ventaja mutua tiene un desarrollo concreto.

El comienzo de una justicia regida por la idea de la ventaja mutua nos obliga a retrotraernos hasta el momento en que se da el paso del estado de naturaleza a la creación efectiva de las sociedades o grupos comunitarios. Este paso surge mediante acciones cooperativas entre las partes participantes que se pueden expresar al estilo hobbesiano, del siguiente modo: los individuos aceptan negociar y someterse a un pacto por el beneficio que éste les reportará. En este contexto, la libertad de intercambio (Barry, 1989b) y a la asunción del mercado como zona moralmente libre (Gauthier, 1986) y garante de los derechos que permiten la libertad de intercambio y negociación adquieren una especial relevancia. En suma, el mercado se entiende como una zona en la que impera la justicia y donde los individuos pueden beneficiarse mutuamente de la cooperación sin tomar ventaja los unos en perjuicio de los otros (ibid.: 113). Es una teoría que, por tanto, conecta la moralidad de los acuerdos a la racionalidad. Sin embargo, no la supedita a ésta en tanto que "para elegir racionalmente, uno debe elegir moralmente" (ibid.: 4$)^{6}$. Si bien pudiera parecer en algún punto contradictorio, la consistencia y coherencia de esta posición descansa en los elementos sobre los que se fundamentan las condiciones que hacen -o debieran hacer- del acuerdo en cuestión uno de tipo racional y moral y que irán apareciendo a continuación.

Este paso del estado de naturaleza a la creación de grupos sociales, a través de acciones cooperativas y del mercado como garante de libertades y zona moral, se establecen las bases de la apropiación de un bien en términos de justicia. En este aspecto, el derecho a un bien apropiado vendrá determinado por las circunstancias en las que dicha apropiación se dé, haciendo legítimas estas situaciones sólo si se deja lo suficiente para los demás y además esto es igual de bueno ${ }^{7}$. Esto es lo que se

\footnotetext{
${ }^{6}$ Las traducciones de lenguas diferentes al español son propias.

7 Por esta idea de "dejar suficiente y tan bueno a los demás", que puede encontrarse en el Second Treatise of Government de Locke (1690). Nozick (1974) lo ha denominado como lockean proviso.
} 
ha Ilamado la condición lockeana (lockean proviso). Brevemente, la finalidad de la condición lockeana -junto con la propuesta que le seguirá de Gauthier sobre los acuerdos racionales- intenta que el mercado se convierta en un espacio común donde los individuos acudan a él en actitud negociadora y cooperante, siendo conscientes de que el bien propio se adquiere bajo los términos de la colaboración entre iguales y sin buscar el beneficio propio mediante el perjuicio de los demás. Este es el sentido del mercado en tanto que zona moral. En lo relativo a las apropiaciones, la condición lockeana nos dice que si una adquisición no empeora la situación de los demás será considerada como justa y se tendrá derecho a ella (Arneson, 1991). Por otra parte, la distribución de tales bienes sólo podrá ser justa si se tiene derecho a ellos, no pudiendo legar o dar nada para lo que no se tiene un legítimo derecho de propiedad.

Finalmente, a fin de corregir posibles injusticias en la distribución de bienes, la medida en que se empeore o mejore algo debe analizarse no calculando cómo sería la situación de otro individuo respecto a ese bien, sino solamente cómo se encontrará ese bien respecto al modo en que uno mismo lo adquirió inicialmente. De lo que se trata es de calibrar el estado del bien a distribuir, no de la persona respecto al bien. De esta manera, podremos afirmar que una distribución será justa si lo que se deja es "suficiente y tan bueno" con respecto a la apropiación original.

Una vez superado el estado de naturaleza mediante estos prerrequisitos fundamentales para garantizar la moralidad y justicia, comienzan los acuerdos racionales cuya finalidad es la búsqueda del mutuo beneficio de los participantes sin que ninguno tome ventaja (de manera injusta) sobre los demás. La complicación, en este aspecto, es cómo hacer de un acuerdo algo justo para los agentes racionales con preferencias opuestas y, por tanto, en disputa. Si lo que nos exige la racionalidad es la maximización de nuestras preferencias, ¿cómo hacerlo sin que eso implique la derrota del prójimo? Es decir, si entendemos que somos racionales en la medida en que buscamos maximizar nuestra función de utilidad y hacer esto conlleva apostar por la máxima ganancia evitando la mayor pérdida posible, ¿de qué manera es posible conciliar esta situación con no pretender la máxima pérdida en el otro? Más aún, ¿es esta actitud compatible con una idea de la ventaja mutua en tanto que acción cooperativa, si ésta se basa en la derrota del otro?

Es en estos términos en los que David Gauthier (1986) desarrolla lo que ha denominado minimax relative concession (MRC). Para evitar que un agente racional vaya predispuesto a maximizar su utilidad a costa de la mayor pérdida del otro puesto que lo que uno gana es directamente proporcional a la pérdida del otro-, el MRC propone que las máximas aspiraciones sean minimizadas y las mínimas concesiones a las que uno está dispuesto a llegar sean maximizadas. Dicho en otras palabras, lo que plantea el MRC es que el nivel de lo que pretendemos conseguir o ganar en una negociación sea reducido para hacerlo aceptable para las otras partes negociadoras. Por otro lado, en cuanto a las concesiones, habríamos de estar dis-

\footnotetext{
${ }^{8}$ Richard Arneson, en cambio, ha dividido esta visión, de Locke, del derecho a un bien en dos condiciones: la de equivalencia y la de no estropear o desperdiciar. Ambas remiten a esa idea lockeana de dejar suficiente (no estropear o desperdiciar) y tan bueno (equivalente) a los demás.
} 
puestos a elevarlas. El resultado sería acudir a una negociación con una actitud más moderada o más humilde en cuanto a aquello que aspiramos a conseguir. De esta manera, los agentes racionales asentarían las bases de sus acuerdos racionales en pretensiones más aceptables para todas las demás partes, bajando el umbral de lo que están dispuestos a ganar y subiendo la línea que delimita lo que están dispuestos a conceder al otro. Lo que en la práctica supone es que se alcance una cierta equivalencia en las dotaciones concedidas y en los beneficios obtenidos. De no existir esta equivalencia beneficio/concesión entre los participantes de la negociación el acuerdo se torna arduo, pues en la misma idea de la condición lockeana se constata que una distribución sólo será justa si se ofrece lo suficiente y tan bueno a lo previamente adquirido. En otras palabras, sin distribuciones equivalentes no hay acuerdo racional posible, sencillamente porque no se da el elemento de la ventaja mutua.

Esta maximización de las concesiones y minimización de la utilidad está fuertemente influida por el equilibrio de Nash (de Jong, 2012: 262), según el cual una situación de equilibrio se define como aquella en la que dos o más agentes racionales no disponen de incentivos para elegir cualquier otra estrategia o curso de acción. Llegar a ese punto de equilibrio requiere de todos los jugadores el previo análisis de las posibles estrategias del resto de jugadores, de modo que ningún individuo configure su curso de acción únicamente según sus preferencias, sino valorando las posibles preferencias y estrategias de los demás. El resultado será que un individuo $A$ adecuará sus acciones en virtud de cómo crea que actuará el resto. En última instancia, puesto que todos deciden jugar la carta de su mejor estrategia -teniendo presente la posible jugada del resto de jugadores- se alcanzará una situación de equilibrio donde ninguno de ellos dispondrá de incentivos para cambiar de estrategia porque, una vez más, esa acción es la que se ha considerado (previo análisis) como la mejor.

Sin embargo, hay una diferencia notable que se sitúa en el punto de partida de cada una de las situaciones hipotéticas que plantean ambos conceptos. Tal diferencia encuentra su fundamento en la constricción de la maximización que ocurre en el punto de salida del MRC. Esta constricción asegura que aquellos que vayan a negociar lo hagan sin la intención de buscar la máxima utilidad individual posible, algo que se encuentra en el corazón mismo del MRC. En el MRC los negociadores parten directamente desde una postura tendente al equilibrio. No se necesita $-\mathrm{O}$ no requiere- de un análisis de las preferencias de los demás, sino que basta con reducir las propias pretensiones y aumentar las concesiones a las que se está dispuesto llegar. De hecho, los agentes racionales, en este caso, nunca abandonan la propia perspectiva ni se someten a un análisis de los bienes del grupo, sino que desde la propia visión de lo que uno prefiere contribuye al bien del grupo y a la consecución de lo que los demás demandan. De modo que se convierte en un principio moral que regula la propia negociación, el intercambio, el establecimiento del mercado en términos morales y, en definitiva, el mutuo beneficio de los participantes (de Jong, ibid.: 262). Es un principio moral que limita la propia acción humana y la búsqueda de la máxima utilidad individual evitando, con ello, posibles abusos. 
En resumen, la teoría de la justicia como ventaja mutua parte de tres premisas. En primer lugar, una concepción que establece cuándo una propiedad privada es adquirida de manera legítima y cómo garantizar una justa distribución de los bienes, desarrollada a través de la condición lockeana. En segundo lugar, un principio que constriñe las exigencias de los individuos, estableciendo la base moral de lo que suponen negociaciones y acuerdos justos, estipulado por el minimax relative concession. Finalmente, la creación y asunción del mercado como una zona moralmente libre, que concede la libertad de intercambio y garantiza el beneficio mutuo de sus participantes, haciéndolos llegar a acuerdos equilibrados y justos y evitando abusos de unos sobre otros de tal manera que, de haberlos, sería imposible en términos racionales establecer ningún acuerdo. El acuerdo exige de racionalidad en las demandas y en lo que se concede. Exige, en última instancia, cierta equivalencia entre lo dado y lo adquirido en un principio (Gosseries, 2001: 303-311).

Ahora bien, la teoría de la ventaja mutua adolece de ciertas debilidades en el contexto intergeneracional. En concreto, esta teoría de la justicia se vuelve muy dependiente de dos circunstancias sin las cuales plantea muchas dudas: el solapamiento generacional, de un lado, y la propia idea del beneficio mutuo, del otro (Fleurbaey y Michel, ibid.: 52-53; Gosseries, 2015: 224-225). Si atendemos a la primera condición, una teoría de la ventaja mutua sólo puede ofrecer una consistencia más o menos fuerte en el caso de que se den generaciones que coincidan en el tiempo: es decir, que se solapen. La idea de generaciones distantes en el tiempo hace que los acuerdos no sólo sean difíciles, sino que, de facto, sean inexistentes. Podríamos alegar una suerte de provisionalidad de los acuerdos hasta su ratificación, enmienda o impugnación futura, pero esto sólo nos lleva a una situación en la que es imposible asumir responsabilidades. En la peor de las situaciones, esto se traduce en una imposición constante de las condiciones contractuales de las generaciones pasadas hacia las futuras, teniendo éstas como única salida la ruptura o reforma del pacto que, del mismo modo, no podrá ser jamás consensuado con las generaciones no nacidas ${ }^{9}$.

De otra parte, esta teoría de la justicia intergeneracional ha de atender al desafío que supone el propio hecho de conseguir una ventaja mutua para todas las generaciones, asumiendo la dificultad del solapamiento generacional. Como se puede ver, este es un reto que se añade en el momento en que nos planteamos tener que llegar a un acuerdo racional con generaciones de un futuro distante. Si tomamos conciencia del impedimento que la propia distancia generacional nos impone para tan sólo negociar, no podemos negar la dificultad añadida de, además, pretender acordar unas condiciones que también resulten favorables para aquellos que ni si quiera han tenido voz para defender sus planteamientos e intereses. En definitiva, la justicia intergeneracional como ventaja mutua debe poder responder de manera consistente al reto que suponen estas dos circunstancias para distribuir de manera

\footnotetext{
9 Esta circunstancia, de hecho, se produce en cuestiones relativas a las reformas constitucionales. Pero que generaciones presentes vivan con un acuerdo constitucional al que llegaron generaciones pasadas no es motivo de injusticia, lo que hace pensar que, quizá, sea otro el enfoque necesario para dar cabida a este tipo de circunstancias.
} 
justa los costes, beneficios y bienestar ascendentes (de una generación a la anterior) y descendentes (de una generación a la siguiente).

\section{LA TEORÍA DE LA SUFICIENCIA}

Como teoría de la justicia intergeneracional, el suficientarismo deriva de un informe que dirigió la ex-primera ministra noruega Go Harlem Brundtland para la World Comission on the Environment and Development (WCED). En el informe, Brundtland propone una definición de desarrollo sostenible, según la cual, éste lo es en tanto que "satisface las necesidades del presente sin comprometer la habilidad de las generaciones futuras para satisfacer sus propias necesidades" (WCED, 1987: 53). En sentido contrario, por tanto, el desarrollo no podrá Ilamarse sostenible si no es capaz de, mientras cubre las necesidades actuales, no poner en peligro las necesidades básicas del futuro. Si nos atenemos a la definición, encontramos que el nexo entre la satisfacción de necesidades y un tipo de desarrollo adecuado es el término sostenible. Podríamos entender que, para poder garantizar las necesidades básicas del futuro, así como las del presente, debemos promover un desarrollo que se caracterice por su sostenibilidad. Dicho de otra forma, en la medida en que se alcance esta sostenibilidad, nuestro futuro -y nuestro presente- estará asegurado. Ahora bien, el término de sostenibilidad no nos indica medida alguna de cálculo de nuestras obligaciones y transferencias intergeneracionales. En cambio, si se presta atención a la definición de Brundtland, es posible percatarse de que dicho concepto de sostenibilidad se caracteriza por ser suficiente para que se produzca el cubrimiento de las necesidades presentes y futuras puesto que, si éstas se producen, el desarrollo al que hace alusión será adecuado para los fines pretendidos. En otras palabras, basta que se produzca un desarrollo sostenible para que este desarrollo sea moralmente deseable. Siendo así, más que centrar el foco en la sostenibilidad habrá que preguntarse qué información distributiva nos da la característica de suficiencia de la que goza el desarrollo sostenible.

El suficientarismo, como doctrina distributiva, podría definirse como aquella teoría que le da al hecho de que ningún individuo caiga por debajo de un umbral -donde puedan sufrir estados de privación severa o pobreza- una gran importancia moral (Casal, 2007). De esta manera, tener lo suficiente importa en términos morales, pues significa disponer lo suficiente para no sufrir algún tipo de privación grave. Hay, no obstante, dos posibles formas de entender la importancia relativa que se le conceda al principio de suficiencia y que pueden ser expresadas mediante dos tesis. De un lado, la tesis positiva ${ }^{10}$, se limita a sostener que mantener a los individuos por encima de esa línea crítica de privación es un valor moral perentorio y, de otro lado, la tesis negativa que argumenta que la suficiencia es lo primordial y único importante moralmente en la lucha contra la desigualdad y, por lo tanto, no necesita de opciones

\footnotetext{
${ }^{10}$ La tesis positiva la abordaré más adelante, en el epígrafe dedicado al igualitarismo rawlsiano por dos motivos: a) por tener un mejor encaje como complemento a otros principios de la justicia y b) porque la formulación del suficientarismo se ha elaborado en su versión negativa principalmente.
} 
distributivas adicionales (Casal, ibid.: 298-304) ${ }^{11}$. Esto se sigue de las palabras de Harry Frankfurt, cuando expresa: lo "que es importante desde un punto de vista de la moralidad no es que todos deban tener los mismo, sino que cada uno tenga suficiente. Si todos tuvieran suficiente, no tendría consecuencias morales si algunos tuvieran más que otros" (Frankfurt, 1987: 21). En estos términos, lo que queda de manifiesto es la consideración de un principio de suficiencia no sólo como expresión de gran importancia en la supresión de estados de privación, sino también como principio excluyente respecto a otros principios distributivos disminuyendo, de esta manera, la importancia que pudieran tener otros requisitos distributivos adicionales. Es el hecho de ser una condición suficiente para producir algo. Es, en los términos expresados por Brundtland, una condición suficiente (el desarrollo sostenible) para satisfacer las necesidades básicas presentes y futuras.

Así planteada, la teoría suficientarista está expresada en términos distributivos, es decir, en su dimensión sustantiva. Una distribución suficiente, de esta manera, corresponde a la cuantía de nuestras obligaciones intergeneracionales caracterizada por ser capaz de satisfacer las necesidades básicas de las personas y situarlas por encima del límite de la pobreza. Esta es su máxima sustantiva, el contenido de las obligaciones que las generaciones deben cumplir. Lo que cabe averiguar ahora es el fundamento de dichos deberes intergeneracionales.

La justificación del surgimiento de obligaciones en el suficientarismo surge como contraposición a la teoría igualitarista, por lo que el rechazo al valor de la igualdad como condicionante de una teoría de la justicia será determinante. No lo niega, empero, aunque sí le resta buena parte de la envergadura de la que le dota el igualitarismo. Los motivos pueden resumirse en tres: el argumento de la relatividad y la comparabilidad, la alienación del individuo y la falta de imparcialidad ${ }^{12}$. En el caso del primer argumento crítico, los autores que apuestan por el suficientarismo o por otras teorías distributivas, señalan que el concepto de igualdad surge de la constante comparación entre personas y entre los recursos de cada una, de la visión de que otro tiene más o menos que yo y que, en virtud de este hecho, yo debo merecer más o menos de algo. Es el hecho de supeditar la caracterización de una mala situación en virtud de que exista otra situación mejor con la que pueda ser comparada (Parfit, 1997). En otras palabras, es hacer dependiente y relativa la calificación de una situación como perjudicial e indigna en función de la existencia de situaciones de vida mejores. Por el contrario, podría replicarse que lo que hace "malvado que unas personas tengan peores vidas no es que otras personas tengan vidas mejores. La

\footnotetext{
${ }^{11}$ El suficientarismo, como doctrina distributiva, ha sido desarrollado especialmente en su tesis negativa, como contraposición a otras teorías de la distribución: el igualitarismo y el prioritarismo. Un primer trabajo desarrollando esta idea de la suficiencia, contrapuesta a los principios de la igualdad y la prioridad, puede verse en Frankfurt (1987). Sobre el prioritarismo, ver Parfit (1997).

${ }^{12}$ En realidad, las dos últimas se siguen de la primera: se aliena por tomar como medida la posición ajena y, por usar también a los otros como baremo para discernir qué tan bien o mal se encuentra un individuo, se rompe con la imparcialidad. No obstante, suponen líneas argumentales distintas.
} 
maldad descansa en el inconfundible hecho de que una vida mala es cruel" (Frankfurt, 1997: 6).

La segunda crítica se sigue directamente de la anterior. El acto de una continua comparación con los demás como forma de determinar qué tan buena o mala es una vida lleva a una alienación del propio individuo, en tanto que sujeto a un conjunto de consideraciones y requerimientos distintos de su propia naturaleza y propias circunstancias personales. Es definir los objetivos vitales única y exclusivamente en función de observaciones externas que no dicen nada de la propia interioridad, necesidad y coyuntura personales. Condiciona, por tanto, el análisis y desarrollo individual mediante factores externos y completamente ajenos (Frankfurt, 1997: 13). Finalmente, la última desavenencia con el igualitarismo respecto del principio de igualdad como principio fundamental de la justicia señala que la naturaleza comparativa de la igualdad hace que falle en el requerimiento de la imparcialidad (Frankfurt, 1997: 11). No se reparte de manera equitativa porque se parta desde una posición neutra, sino que reparte equitativamente dependiendo de que otros tengan mayor o menor cantidad de algo.

La contraposición al igualitarismo refleja un claro desacuerdo respecto a aquello que podemos considerar como valor primordial para una adecuada concepción de la justicia. En el caso de los suficientaristas, el valor fundamental podríamos encontrarlo en el respeto. Este respeto se distinguiría de la idea de la igualdad en tanto que no es simplemente una cuestión de proveer a cada persona de lo mismo que tienen las demás. El respeto es algo de carácter personal desde donde se atiende a los individuos "exclusivamente sobre la base de aquellos aspectos de sus características particulares o circunstancias que son realmente relevantes al problema en cuestión" (Frankfurt, 1997: 8) y que, por tanto, es más fundamental que el principio de igualdad. Una lectura atenta descubrirá que este principio normativo se postula como oposición a esa naturaleza relativa que se le imputa a la igualdad, por lo que el respeto sería la razón oportuna para nutrir a una concepción de la justicia de una verdadera imparcialidad. Es a consecuencia de este respeto -que le debemos a todos los seres humanos y que procede de características de la propia naturaleza humana o de la racionalidad-por lo que está dentro de nuestras obligaciones tratar de manera equitativa a todos los individuos realizando distribuciones suficientes para satisfacer las necesidades básicas de éstos. Sólo en la medida en que se ponga en valor el respeto hacia todas las personas podremos hacer efectiva unas distribuciones (de recursos, oportunidades, bienestar, etcétera) imparciales que aseguren que nadie pueda caer en la pobreza o ser gravemente discriminado. De esta manera, el respeto constituye la máxima justificadora de la teoría suficientarista.

Sin embargo, el primer revés a esta teoría va dirigido a esa idea según la cual el igualitarismo encierra un carácter profundamente relativo y sujeto a la constante comparación entre individuos. El argumento de la discriminación analiza si la teoría de la suficiencia puede dar buena cuenta de los casos en lo que se discrimina. Si la igualdad se muestra abiertamente parcial, la noción de respeto -por atender a una característica común a todas las personas- debería poder distinguir aquellos casos donde se den hechos de flagrante discriminación respecto de aquellos menos lesivos. Sin embargo, el concepto del respeto como principio normativo no nos proporciona 
las herramientas apropiadas para distinguir un caso discriminatorio grave de uno irrelevante en términos morales (Casal, ibid.: 302-303). La teoría de la suficiencia parte de la presunción de que atender a una comparación entre individuos implica necesariamente establecer lo que es bueno o malo, justo e injusto, en función de si hay individuos mejor que otros, de tal manera que caracterizaríamos una situación privativa por mostrarse claramente peor que otra donde esto no se da. Por tal consideración, el respeto debería ser suficiente para mantener la justicia. En cambio, una lectura razonable de las tesis igualitaristas rechaza cualquier postura que se aproxime a lo que el suficientarismo le imputa ${ }^{13}$.

La noción de respeto puede defender cualquier tipo de desigualdad, pero no alberga en su contenido normativo una sola cláusula que nos diga por qué determinados tipos de discriminación requieren de una mayor atención que otros. Para cualquiera de los casos, el principio de respeto mide la injusticia en virtud de una asimetría entre el trato dado a un individuo y el trato que merece a causa de su humanidad o racionalidad, pero no alcanza a ver qué factores concretos se ven comprometidos. A fin de cuentas, una discriminación por causas raciales ataca tanto al respeto que merecen las personas por el hecho de ser personas como una exclusión por superstición que pudiera llevar a cabo el dueño de un establecimiento comercial al no atender los días martes al cliente número trece por orden de llegada. Y, sin embargo, la primera de ellas parece de mayor gravedad que la segunda.

La segunda objeción a la máxima justificadora es complementaria a la anterior. Si el hecho de comparar situaciones propias con ajenas (en cuanto a recursos, oportunidades, niveles de bienestar) verdaderamente supusiera una alienación del individuo, habría que concluir, como ya lo hiciera Robert Goodin (1987: 44-49), que entonces sería igual de malo prestar atención a cualquier cosa que hiciera de la vida algo más llevadero, como optar por un salario mejor o maximizar nuestra salud. Al fin y al cabo, no dejan de ser elementos externos. Además, es posible que nuestras intuiciones morales nos digan que siempre será mejor un mundo en el que podamos preguntarnos cuál es la causa de que unos tengan tan poco en comparación con otros. En este sentido, quizá tendamos a pensarnos como iguales de manera previa a la posesión de un estatus o bienes determinados. Finalmente, el suficientarismo encuentra problemas en el escenario distributivo (Casal, ibid.: 307-308, 315-317; Wolf, 2007: 292). Al no permitir otras opciones distributivas adicionales, lo que ocurra por encima de la línea de la suficiencia deja de importarle y no tiene en cuenta que dentro de cada generación puede haber miembros en desventaja y que puede ser más urgente una redistribución intrageneracional que limitarse a las transferencias intergeneracionales. En definitiva, cabe sospechar que la suficiencia no llega a ser suficiente.

\section{EL IGUALITARISMO RAWLSIANO}

Probablemente, la teoría más compleja de todas sea el igualitarismo en los términos en los que John Rawls lo expuso en su gran obra $A$ Theory of Justice. Una de las diferencias más notables frente al resto de teorías de la justicia intergeneracional es que Rawls no cierra la discusión de la justicia en torno a un único principio. El filósofo

\footnotetext{
${ }^{13}$ Posibles respuestas a esta cuestión pueden encontrarse en Goodin (1987) o Temkin (2003).
} 
norteamericano fue consciente de que el sentido de la igualdad no bastaba para dar cumplimiento de nuestras percepciones de la justicia. No todo debe ser igualado, ni todas las personas merecen llevar una vida igual a los demás. La teoría rawlsiana, además de establecer la importancia del concepto de igualdad, concede un papel predominante a la libertad individual. Por lo que no sólo hablamos de una teoría igualitarista de la justicia, sino también liberal. Pero aún es más, el autor supo ver la necesidad de dar cabida a criterios de suficiencia o de maximización ${ }^{14}$, lo que dota a su teoría de la justicia de una estructura de mayor riqueza y de gran interés. Adelantándose al grueso de las críticas realizadas a la doctrina del igualitarismo, la virtud del enfoque rawlsiano puede resumirse en la recomendación que Samuel Scheffler (2005) hacía a los liberal-igualitaristas, y es que éstos tienen que concebir la equidad no sólo como un principio distributivo, sino como un principio normativo que responda a la visión de una sociedad de iguales.

Rawls (ibid.: 47-101) resume en dos los principios de la justicia: el de igual libertad y el de diferencia. El primero de ellos exige que una concepción de la justicia garantice el derecho de la igualdad en cuanto a libertades de todos los individuos. Por otro lado, el segundo constata que las desigualdades socioeconómicas son tolerables en la medida en que éstas vayan dirigidas al beneficio de los menos aventajados de la sociedad. Así, en estos dos principios Rawls establece la máxima justificadora y la máxima sustantiva: cualesquiera que sean nuestras obligaciones, éstas derivan de una consideración en virtud de la cual lo que es justo viene definido por la igual provisión de libertades a todos los individuos y, además, la obligación de corregir las posibles situaciones de desventaja de aquellos que se encuentren en una peor situación en nuestra sociedad, de lo que se deriva la equidad a la que deben responder las distribuciones en derechos y libertades.

El modo de llegar a estos principios de justicia viene determinado por la posición original y el velo de ignorancia (Rawls, ibid.: 102-170). Brevemente, el autor crea una situación hipotética en la que, a fin de asegurar la imparcialidad en la concepción de la justicia, agentes racionales han de escoger los principios normativos que regirán la vida en sociedad desde cero y en la que estos agentes racionales se encontrarían desprovistos de información sobre cuál será su situación final, cuál será su nivel de bienestar, a qué escala social pertenecerán y, en definitiva, de todo conocimiento indicativo de una posición determinada en la sociedad. Bajo un punto de vista utilitarista, un jugador racional bien podría optar por aquellos principios que maximicen la utilidad general presuponiendo que una mayor utilidad para el conjunto de los ciudadanos implica una mayor cantidad a repartir -aunque éste conlleva también un mayor riesgo- ${ }^{15}$. Sin embargo, el error está en suscribir esa idea de la racionalidad.

\footnotetext{
${ }^{14}$ También podría hablarse del utilitarismo como teoría de la justicia intergeneracional, pero para este trabajo lo descarto por dos motivos: primero, es una teoría de carácter agregativo, más que distributivo; segundo, el enfoque rawlsiano ya contiene criterios utilitaristas como la fase de acumulación en la estructura intergeneracional.

${ }^{15}$ Un pensamiento similar es el que presupone -con razón o no- que un mayor porcentaje de PIB en un país supone mayor riqueza repartida para todos los ciudadanos que forman dicho país.
} 
Concretamente, la irracionalidad remanente en este tipo de cálculo racional es concebir la formación de las sociedades y sus principios de justicia como una situación de juego clásico, donde el reparto equitativo se produce probabilísticamente "mano tras mano". En tal caso, un jugador, tiene las mismas varias oportunidades de recuperar un mal reparto inicial. Muy al contrario, en la posición original el reparto se produce una y sólo una vez en la vida, por lo que no caben más repartos posibles (Waldron, 1986: 25-26). A fin de cuentas, uno sólo puede nacer una vez. "Desde que el acuerdo original es definitivo y hecho a perpetuidad, no hay segunda oportunidad" (Rawls: ibid.: 153).

Tenga razón o no John Rawls es posible pensar que los individuos desarrollarán una cierta aversión al riesgo que les hace apostar por una opción más segura: una sociedad igualitaria donde todos partan con los mismos derechos y libertades para poder desarrollar su vida (principio de igual libertad) y que, tras el respectivo reparto de las posiciones en la sociedad, se configure un seguro que no deje en el olvido a quienes han gozado de peor suerte en la distribución inicial (principio de la diferencia). No obstante, haríamos mal en concebir el argumento rawlsiano como uno meramente de elección racional. Más bien, el planteamiento de Rawls es uno de tipo moral (Waldron, ibid.: 26). La pregunta que se les hace a los agentes racionales en la posición original no es qué tipo de principios quieren, pues esto derivaría en una multiplicidad difícilmente conjugable de preferencias particulares, sino que la pregunta sería: “¿con qué principios estaríais dispuestos a comprometeros?" Por lo tanto, ya no es una situación de mera elección racional, sino de compromiso. Es un escenario de palabra dada, de obligaciones contraídas.

Por su parte, el principio de diferencia incorpora algunos elementos suficientaristas de gran interés. Una lectura atenta de los dos principios de justicia bien podría suponer que -en clave de derechos y libertades- el principio de la igual libertad está pensado no sólo en tanto que noción de igualdad, sino también de suficiencia. En materia constitucional, podríamos señalar que la igualdad en derechos y libertades es una medida de suficiencia destinada a garantizar, precisamente, la igualdad. Este social minimum (Rawls, 1999: 244-245, 251-252, 278-280; 2001: 47-48) juega el papel de un seguro que refuerza el principio de diferencia a lo largo de toda la vida del individuo (Van Parijs, 2002). Es decir, si el principio de diferencia está ideado como una fuente de redistribución de los recursos en favor de los más desaventajados, el social minimum ayuda a asegurar que la distribución de las libertades no sea meramente formal, sino que sean efectivamente disfrutadas por todos los ciudadanos (Rawls, 2005: 324-331). En definitiva, configuran las "medidas tendentes a asegurar a todos los ciudadanos medios adecuados para cualquier fin que les permitan hacer uso efectivo de sus libertades y oportunidades básicas" (Rawls, 1994: 13). La complementación, por tanto, del principio de diferencia y del social minimum, más allá de ser redundantes, constituyen una útil manera de equilibrar los valores centrales una teoría de la justicia entendida como equidad (Casal, ibid.: 324$)^{16}$.

\footnotetext{
${ }^{16}$ En este aspecto es el que tiene cabida la tesis positiva del suficientarismo. La misma no sólo expone la gran relevancia de una medida de suficiencia, sino que deja abierta la posibilidad de complementarse a otros principios distributivos. Es interesante este elemento porque
} 
En cuanto al ámbito intergeneracional, Rawls establece una estructura específica para tratar este dominio de la justicia. En este contexto, surge el problema de que generaciones demasiado jóvenes o nonatas sean meros receptores sin capacidad ni posibilidad alguna de incidencia en las decisiones de lo que heredarán, por lo que cabe esperar que estas generaciones estarán peor que las actuales si éstas últimas no hacen nada por evitarlo. En este sentido, la estructura de las demandas de justicia intergeneracional se divide en dos estadios: un primer período de acumulación y un segundo de estabilidad (Gosseries, 2001, 2004, 2005, 2015; Rawls, 1999: 251-258). La primera fase responde a criterios fundamentalmente utilitaristas en lo que respecta a las distribuciones. Mientras que, en la segunda fase, vuelven a prevalecer principios distributivos igualitaristas. La pretensión con esta doble estacionalidad intergeneracional es la de establecer un principio del justo ahorro, el cual pueda garantizar en qué momentos es requerido ahorrar para asegurar los derechos, libertades e instituciones básicas de una sociedad justa y en qué momentos el ahorro intergeneracional no es obligatorio. De forma sucinta, la fase de acumulación exige un determinado nivel de ahorro para asegurar los requerimientos fundamentales de la sociedad justa y la fase estable elimina el deber de ahorrar puesto que ya estarían aseguradas esas libertades e instituciones justas de las que hablo (Rawls, 1999: 255) ${ }^{17}$. La aparición de dos estadios en el contexto intergeneracional es, ciertamente, algo novedoso entre las teorías que ya se han expuesto.

En los términos planteados, el marco intergeneracional del igualitarismo de Rawls parece ajustarse bastante bien a las derivas socioeconómicas de los países. En épocas de transición hacia un modelo laboral o de seguridad social distinto del actual, que pueda acarrear costes, se hace imprescindible contar con ahorros para facilitar y afianzar ese cambio. De hecho, el paso de la fase acumulativa a la estable no es otra cosa que una transición hacia una organización, disposición y constatación de los derechos, libertades e instituciones que la sociedad justa requiere. En última instancia, la composición de los dos estadios intergeneracionales es una medida orientada a dar cumplimiento a los principios de igual libertad y diferencia. Es curioso, con todo, que la etapa a la que le es asignada ser garante de ambos principios sea la confeccionada con criterios utilitaristas. Pero lo certero del enfoque rawlsiano es establecer seguidamente una fase estable donde los principios utilitaristas no son necesarios.

Personalmente, considero que el enfoque rawlsiano dispone de una posición normativa más elevada que las teorías antes expuestas por varios motivos. Contrario a tesis suficientaristas, no basamos la justicia en el respeto a las personas, sino en estimar que lo que es justo viene definido por una igual apreciación respecto a los derechos y libertades que merecemos y necesitamos para la correcta vida en sociedad.

transforma de alguna forma el carácter del principio de suficiencia. Deja de ser una condición meramente suficiente para ser una de tipo necesaria y suficiente. Así formulado, el social minimum tiene un encaje bastante atractivo en la teoría igualitarista rawlsiana en tanto que condición necesaria y suficiente sin el cual no hay posibilidad de conseguir la igualdad y cuya existencia basta para afianzar las libertades básicas.

17 Este ahorro debe interpretarse en clave maximizadora (al estilo utilitarista) donde de contribuye a una mayor "cesta" de recursos. 
Es una perspectiva que atiende a razones individuales, pero cuyo objetivo está centrado en la regulación de la sociedad. Por otro lado, al contrario que en la ventaja mutua, la elección de los principios normativos de la sociedad justa no está basada en un mero planteamiento de elección racional y, también a diferencia de una teoría de la reciprocidad, el igualitarismo liberal de Rawls puede dar sentido al surgimiento de las obligaciones de una hipotética primera generación, haciéndolas recaer éstas en argumentos morales.

Además, es posible destacar el parecido que existe entre la creación de un límite de privación o desigualdad bajo el cual no debe permitirse caer a las personas -propio del suficientarismo- con ese momento en que son alcanzadas las garantías de derechos e instituciones justas del desarrollo de Rawls, que funciona también a modo de límite. Si atendemos a la definición que el mismo ex-profesor de Harvard nos ofrece de la fase de acumulación, ésta debe conseguir "una base material suficiente para establecer instituciones justas efectivas dentro de las cuales las libertades básicas puedan ser todas disfrutadas" (Rawls, ibid.: 256) ${ }^{18}$. Ciertamente, esta definición no difiere demasiado respecto de la doctrina de la suficiencia sostenida por Frankfurt $(1987,1997)$ en tanto que ambas convergen en el establecimiento de un umbral que permita discernir cuándo los individuos están por debajo de aquello que la justicia requiere. Sin embargo, el planteamiento de Rawls parece superior al de Frankfurt en, al menos, un punto. Uno de los problemas asociados a la teoría suficientarista reside en el lugar en que deba establecerse ese límite de suficiencia. Existen dudas acerca de las facilidades de los suficientaristas a la hora de medir el punto en el que establecerlo de manera más o menos precisa. En este aspecto, la virtud del principio del justo ahorro es que no depende únicamente de intuiciones acerca de dónde debe encontrarse ese límite -como ocurre en el suficientarismo-, sino que depende directamente de previsiones y datos empíricos sobre los requisitos materiales para respaldar instituciones liberales sostenibles (Casal, ibid.: 316, 324-325).

Finalmente, una debilidad en el enfoque intergeneracional de la justicia de John Rawls debe ser advertido. Si bien uno de los puntales del liberal-igualitarismo rawlsiano es la atención que dedica la justicia a aquellos más desfavorecidos de la sociedad a causa de un infortunado reparto inicial, ésta no se evidencia del todo en el ámbito intergeneracional y tiene que ver con uno de los pasajes en torno a los que gira la discusión en su $A$ Theory of Justice. Allí concretamente, Rawls afirma lo siguiente: "una vez que las instituciones justas están firmemente establecidas y se cumplen todas las libertades básicas, la acumulación neta requerida cae a cero. En este punto, una sociedad cumple con su deber de justicia al mantener instituciones justas y preservar su base material. El principio de ahorro justo se aplica a lo que una sociedad debe ahorrar como una cuestión de justicia. Si sus miembros desean ahorrar para otros fines, ese es otro asunto" (Rawls, ibid.: 255) ${ }^{19}$. El inconveniente de esta última frase es que, implícitamente, otorga validez al ahorro de aquellas generaciones que se encuentran en la fase estable. De acuerdo al principio de libertad evidentemente se trata de un asunto que bien debiera concernir a dicha generación concreta,

\footnotetext{
${ }^{18}$ Las cursivas son mías.

${ }^{19}$ Cursivas añadidas.
} 
pero permitir el ahorro en ese estadio también puede traer deficiencias redistributivas y, con ello, injusticias a nivel intrageneracional (Gosseries, 2001, 2004, 2015). Con todo, no es una cuestión cuya resolución sea sencilla, pero es importante reseñar que uno de los puntos que diferencian la teoría de la justicia de Rawls respecto de otras es -de manera coherente e inteligente- introducir elementos de carácter redistributivos -como el principio de diferencia-para afianzar una igualdad real y efectiva como motor para que cada ciudadano pueda escoger el modo de vida buena que desea llevar.

\section{CONCLUSIONES}

Lo realizado hasta aquí no ha sido sino el intento de esclarecer en alguna medida cuestiones relativas al marco de la justicia intergeneracional. En especial, el trabajo ha ido dirigido a desgranar las razones que podemos encontrar que justifiquen que tengamos algún tipo de deuda o deber con las siguientes generaciones, además de establecer los principios distributivos pertinentes para cada caso. Si bien la justicia entre generaciones implica la preocupación por generaciones que no tendrán incidencia en nuestra vida, adoptar una determinada concepción de la justicia intergeneracional sí se postula como potencial transformador de nuestro modo de vida en la medida en que, en función de la postura que se adopte, el tipo y contenido de obligaciones de unas generaciones con otras variará de manera significativa.

En este sentido, he analizado cuatro teorías de la justicia entre generaciones. La reciprocidad indirecta descansa sobre razones contractuales de las que se encuentran en la formalización de una deuda. Así, lo que una generación actual debe a la siguiente viene definido por aquellas transferencias que realizara la generación anterior a la presente. El modo de solventar esa deuda, por lo tanto, revista un carácter indirecto. En cuanto a la teoría de la ventaja mutua, su fundamento se caracteriza por estar compuesto por un escenario de negociación y pacto entre distintas generaciones donde prevalece la libertad de intercambio y en el que los agentes racionales disponen de los elementos morales necesarios - a través del mercado- para negociar en pie de igualdad y en busca del beneficio mutuo. El suficientarismo, por su parte, se justifica por el respeto que le debemos a las personas en virtud de su humanidad o racionalidad y que exige que éstas no caigan en estados de discriminación o privación severa. De tal manera, lo que una teoría de la justicia debe proveer es que los individuos dispongan de lo suficiente para que tengan garantizado ese respeto que se les debe. Finalmente, el igualitarismo rawlsiano, en su complejidad, se presenta como una doctrina que asienta sus pilares en los principios de igualdad y libertad, un principio redistributivo como es el principio de diferencia y un social minimum cuya misión es fortalecer los principios de igualdad y libertad, haciéndolos necesarios y suficientes para la correcta vida en sociedad. Por otra parte, los estadios de acumulación y de estabilidad en el contexto intergeneracional hacen que su teoría liberal-igualitaria se adapte mejor a la realidad política y socioeconómica al poder combinar principios utilitaristas e igualitarios. 


\section{REFERENCIAS BIBLIOGRÁFICAS}

ARNESON, R. (1991). "Lockean Self-Ownership: Towards a Demolition", Political Studies, vol. XXXIX, pp. 36-54.

BARRY, B. (1989a). "Justice as Reciprocity", en Liberty and Justice, Oxford: Oxford University Press.

— (1989b). Theories of Justice, Berkeley: University of California Press.

CASAL, P. (2007). "Why Sufficiency Is Not Enough", Ethics, 117 (2), pp. 296-326.

DE JONG, J. (2012). Rethinking Rational Choice Theory: A Companion on Rational and Moral Action, Hampshire: Palgrave Mcmillan.

FLEURBAEY, M. Y MICHEL, PH. (1992). "Quelle justice pour les retraites?", Revue d'économie financière, 23, pp. 47-64.

FRANKFURT, H. (1987). "Equality as a Moral Ideal", Ethics, 98, pp. 21-43.

— (1997). "Equality and Respect", Social Research, 64, pp. 3-15.

GAUTHIER, D. (1986). Morals by Agreement, Oxford: Clarendon Press.

GOODIN, R. (1987). "Egalitarianism: Fetishistic or Otherwise", Ethics, 98, pp. 44-49.

GOSSERIES, A. (2001). "What Do We Owe the Next Generation(s)", Loyola of Los Angeles Review, 35, 293-354.

— (2004). Penser la Justice entre les générations: De l'affaire Perruche à la réforme des retraites, Paris: Collection Alto, Flammarion.

- (2005). "The Egalitarian Case Against Brundtland's Sustainability", GAIA, 14/1, pp. $40-46$.

- (2015). "Teorías de la justicia intergeneracional: Una sinopsis", Revista Jurídica de la Universidad Autónoma de Madrid, vol. II, 32, pp. 217-237.

NOZICK, R. (1974). Anarchy, State, and Utopia, New York: Basic Books.

PARFIT, D. (1997). "Equality or Priority?, Ratio (new series), X, pp. 203-221.

RAWLS, J. (1994). "La idea de razón pública", Isegoría, 9, pp. 5-40.

- (1999). A Theory of Justice, Revised Edition, Cambridge: Harvard University Press.

- (2001). Justice as Fairness: A Restatement, Cambridge: Cambridge University Press.

— (2005). Political Liberalism, New York: Columbia University Press.

SCHEFFLER, S. (2005). "Choice, Circumstance and the Value of Equality", Politics, Philosophy and Economics, 4 (1), pp. 5-28.

SCHOKKAERT, E. Y VAN PARIJS, PH. (2003). "Social Justice and the Reform of Europe's Pension Systems", Journal of European Social Policy, 13 (3), pp. 245-263.

TEMKIN, L. (2003). "Egalitarianism Defended", Ethics, 113 (2003), pp.764-782. 
VAN PARIJS, PH. (2002). "Difference Principles", en Cambridge Companion to Rawls, ed. Samuel Freeman, Cambridge: Cambridge University Press.

WALDRON, J. (1986). "John Rawls and the Social Minimum", Journal of Applied Philosophy, 3: 1, pp. 21-33.

WOLF, C. (2007). "Intergenerational Justice" en Frey, R. G. y Wellman, Ch. eds. (2007), A Companion to Applied Ethics, Oxford: Blackwell Publishing Ltd, pp. 279294.

WORLD COMISSION ON THE ENVIRONMENT AND DEVELOPMENT (WCED) (1987). Our Common Future, Oxford/New York: Oxford University Press. 